\title{
Density Scaling of the Structural and Johari-Goldstein Secondary Relaxations in Poly(methyl methacrylate)
}

\author{
R. Casalini* and C. M. Roland* \\ Naval Research Lab, Chemistry Division, Code 6120, Washington, D.C. 20375-5342, United States
}

ABSTRACT: Dielectric spectra were obtained on a low molecular weight poly(methyl methacrylate) (PMMA) over a range of temperatures $(331<T(\mathrm{~K})<386)$ at pressures approaching $0.8 \mathrm{GPa}$. The $\alpha$ relaxation times, $\tau_{\alpha}$, superpose when plotted versus $T / \rho^{\gamma}$, where $\rho$ is density and $\gamma$ a material constant, in accord with results for many other van der Waals liquids and polymers. However, the Johari-Goldstein (JG) relaxation times, $\tau_{\mathrm{JG}}$, do not conform to this density scaling for the same value of the exponent $\gamma$. Likewise, the frequency separation of the $\alpha$ and JG loss peaks in the spectrum increases with pressure for constant $\tau_{\alpha}$; that is, state points having the same $\alpha$ relaxation time and same peak breadth have different $\tau_{\mathrm{JG}}$. Similar results were obtained on a lower molecular weight

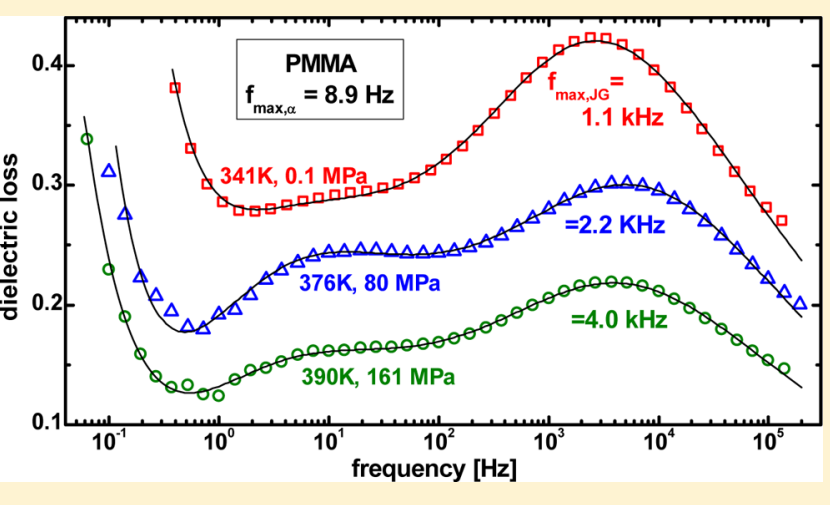
PMMA, for which there was less overlap of the two peaks. The implication is that density scaling of the segmental relaxation times originates in the glass transition dynamics, not, as recently suggested, in higher frequency secondary processes.

\section{INTRODUCTION}

Density scaling refers to the superpositioning of structural relaxation times, $\tau_{\alpha}$, when expressed as a function of the ratio of temperature to density, where the latter is raised to the power $\gamma$

$$
\tau_{\alpha}=f\left(T / \rho^{\gamma}\right)
$$

Equation 1 has been shown to apply to experimental measurements on virtually all nonassociated liquids and polymers. ${ }^{1-6}$ Equivalent relations describe the viscosity, diffusion constant, and other dynamical properties, although when data extend to high temperatures, as common for viscosities $^{7,8}$ and molecular dynamics simulations (mds), ${ }^{9,10}$ the use of scaled quantities improves the superpositioning. ${ }^{11}$ Beyond a means to categorize and organize experimental data spanning broad ranges of temperature and pressure, interest arises in density scaling because of the physical meaning that may be ascribed to the material constant $\gamma$. For example, from mds it has been shown that $\gamma(\mathrm{i})$ is approximately one-third the effective slope of the intermolecular repulsive potential, ${ }^{9,12}$ (ii) equals the proportionality constant between isochoric equilibrium fluctuations of the virial and potential energy, ${ }^{10,13}$ and (iii) from the latter can be connected to linear thermoviscoelastic constants. ${ }^{14}$

Density scaling is invariably applied to $\tau_{\alpha}$ or other dynamical quantities that are coupled to the $\alpha$-relaxation time such as the viscosity and diffusion constant. However, there are two relaxation processes that, while not directly related to structural relaxation, are considered to bear a relationship to $\tau_{\alpha}$ : the terminal relaxation giving rise to a low-frequency dispersion in the mechanical loss of polymers and the Johari-Goldstein (JG) secondary relaxation found in all molecular liquids and polymers. The former is responsible for the normal mode peak in the dielectric loss of polymers having a dipole moment parallel to the chain backbone, such as 1,4-polyisoprene, ${ }^{15}$ polyoxybutylene, ${ }^{16}$ and poly(propylene glycol). ${ }^{17}$ Since theories of polymer dynamics such as the Rouse and reptation models $^{18,19}$ posit that chain motions are governed by the same local friction coefficient associated with the segmental dynamics, superpositioning of the normal mode relaxation time should yield essentially the same value of the scaling exponent found for $\tau_{\alpha}$. To a good approximation, this correspondence is borne out by experimental data. ${ }^{15-17,20}$

The JG relaxation refers to local, noncooperative dynamics not requiring intramolecular degrees of freedom. The JG peak in the relaxation spectrum occurs at the lowest frequency of any secondary processes, and the fact that it precedes in time the $\alpha$ relaxation has been taken to imply that the JG process plays a role in structural relaxation, even serving as its precursor. ${ }^{21}$ This in turn leads to the expectation that JG relaxation times, $\tau_{\mathrm{JG}}$, should superpose for the same scaling exponent as $\gamma$ for $\tau_{\alpha}$. This idea was recently tested using dielectric measurements on diglycidyl ether of bisphenol A. ${ }^{22}$ Most of the JG relaxation data in that work were for temperatures below the glass transition, where changes in $\tau_{\mathrm{JG}}$ with pressure are small (ca. 1 decade or less $\left.^{22}\right)$. Direct determination of $\alpha$ relaxation times in the glass are not possible, and in fact it is currently unknown whether density scaling is valid in the glassy state. Moreover, JG relaxation times in the glassy state are dependent on the thermodynamics path followed during the glass formation; that

Received: June 12, 2013

Revised: July 11, 2013

Published: July 24, 2013 
is, glasses cooled under different pressures can exhibit very different $\tau_{\mathrm{JG}}{ }^{23-25}$ This makes problematic (if not untenable) the hypothesis that $\tau_{\mathrm{JG}}$ are only a function of $T$ and $\rho$ in the glassy state. Nevertheless, the conclusion was drawn in ref 22 that $\tau_{\mathrm{JG}}$ superpose for the same value of $\gamma$ that superposes $\tau_{\alpha}$ when these relaxation times are plotted versus $T / \rho^{\gamma}$. The simultaneous density scaling of the $\alpha$ and JG relaxations was taken as confirmation of a prediction of the coupling model, ${ }^{26,27}$ that these respective relaxation times are related according to ${ }^{21}$

$$
\tau_{\alpha}=\left[t_{\mathrm{c}}^{\beta_{\mathrm{KWW}}-1} \tau_{\mathrm{JG}}\right]^{1 / \beta_{\mathrm{KWW}}}
$$

in which $t_{\mathrm{c}}(\sim 1 \mathrm{ps})$ is a constant and $\beta_{\mathrm{KWW}}$ represents the stretch exponent of the Kohlrausch-Williams-Watts correlation function for the $\alpha$ process. ${ }^{28}$ According to eq 2, if $\beta_{\mathrm{KWw}}$ is constant or a function of $\tau_{\alpha}{ }^{29,30}$ both relaxation times should conform to density scaling with equivalent values of $\gamma$; otherwise stated, the ratio $\tau_{\mathrm{JG}} / \tau_{\alpha}$ is a constant for any value of $\tau_{\alpha}$ for a material conforming to density scaling.

In this paper we describe dielectric measurements on a low molecular weight poly(methyl methacrylate) (PMMA) obtained over a range of temperatures and pressures. Using the equation of state for the material, we express $\tau_{\alpha}$ and $\tau_{\mathrm{JG}}$ as a function of the scaling variable $T / \rho^{\gamma}$. We find that, in accord with results for many glass-forming materials, ${ }^{1-6}$ the $\alpha$ relaxation times superpose according to eq 1 with $\gamma=1.94$. There is a prominent secondary relaxation in the spectra of PMMA that has been identified as a Johari-Goldstein process, that is, involving all atoms of the repeat unit. ${ }^{31}$ We find that $\tau_{\mathrm{JG}}$ for PMMA does not conform to the scaling relationship using the value of $\gamma=1.94$. We also find that for state points having a constant value of $\tau_{\alpha}$ the $\tau_{\mathrm{JG}}$ are not equal. From these experimental observations we conclude that the relation between $\tau_{\alpha}$ and $\tau_{\mathrm{JG}}$ is not invariant; this implies that the coupling model interpretation of density scaling is only approximate, if not incorrect.

\section{EXPERIMENTAL SECTION}

The PMMA (weight-average molecular weight = $1970 \mathrm{Da}$; polydispersity $=1.14$ ) was obtained from Polymer Standard Service, Inc., and used as received. We also included data from measurements on a lower molecular weight (= $402 \mathrm{Da})$ PMMA. For the dielectric measurements the sample was pressed as a melt between two electrodes (diameter $=16 \mathrm{~mm}$ ) separated by a Teflon spacer (thickness $=100 \mu \mathrm{m})$. For the equation of state $(P V T)$ measurements, a $\sim 0.8 \mathrm{~mL}$ plug was molded under vacuum. The density at atmospheric pressure and temperature was measured using the buoyancy method, with the relative changes in density as a function $T$ and $P$ then determined using a Gnomix apparatus with mercury as the confining fluid.

Dielectric spectra were measured with a Novocontrol Alpha analyzer. For measurements at high pressure, the sample was maintained in a custom-built chamber based on a Harwood Engineering pressure vessel, placed in an Tenney environmental chamber (temperature control $= \pm 0.1{ }^{\circ} \mathrm{C}$ ).

\section{RESULTS AND DISCUSSION}

Figure 1 shows representative dielectric constant and loss spectra for the PMMA. Two overlapping peaks, corresponding to the primary $\alpha$ and JG secondary relaxations, are evident in the spectra, along with a contribution toward lower frequencies from ionic conduction. The overlapping worsens at higher temperatures and lower pressures because not only are the peaks closer but also the relative intensity of the JG process

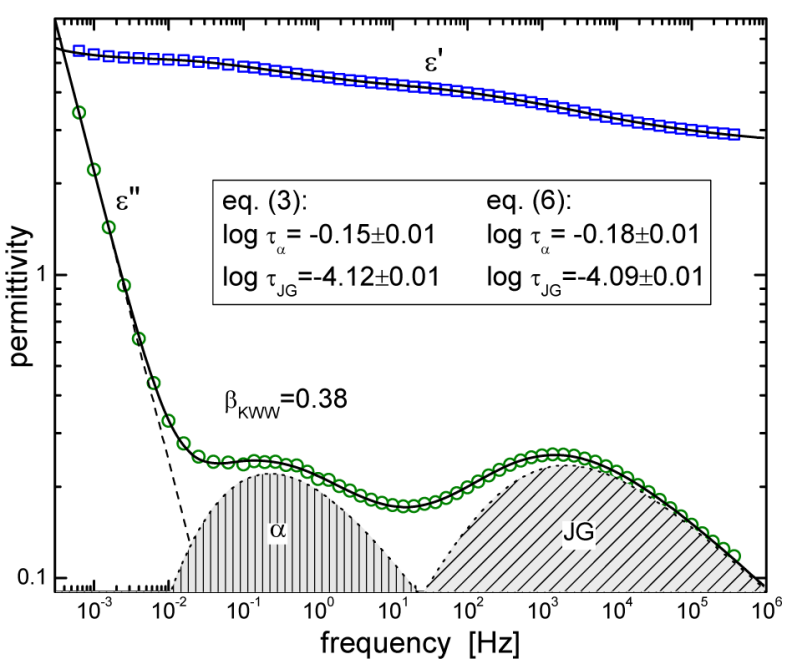

Figure 1. Real and imaginary part of the dielectric permittivity versus frequency for PMMA at $T=360.9 \mathrm{~K}$ and $P=53.2 \mathrm{MPa}$. The solid lines are fits of both eq 3 and eq 6; the difference is not discernible. The contributions of the $\alpha$ and JG processes, along with the ionic conductivity, are indicated.

grows as that of the $\alpha$-relaxation diminishes. This is shown in Figure 2 in a plot of the respective dielectric strengths versus

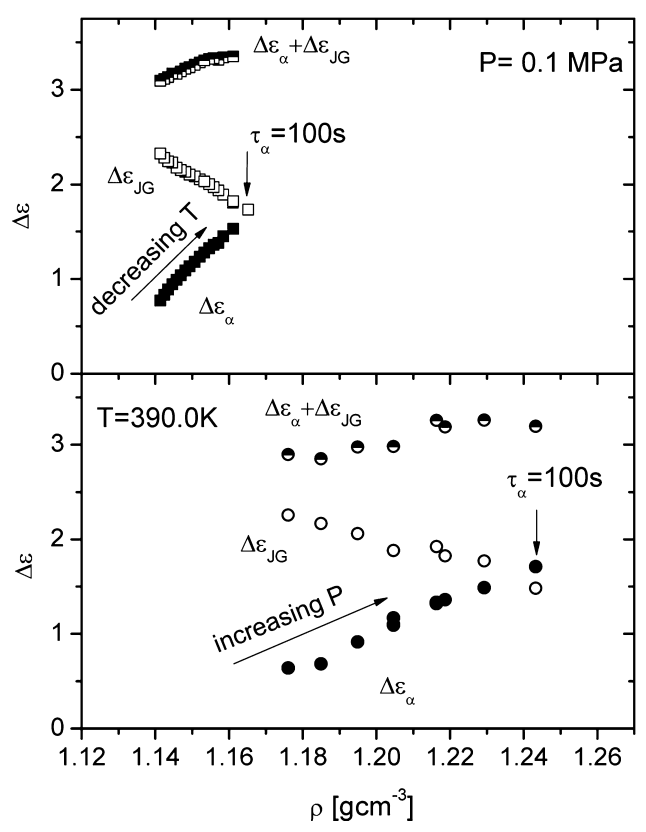

Figure 2. Dielectric strength versus density during isobaric and isothermal measurements for the $\alpha$ relaxation (filled symbols), the JG process (open symbols), and their sum (half-filled symbols).

density, the latter determined for each state point using the equation of state for PMMA (described below). Because of this overlapping, deconvolution of the peaks is required to determine the relaxation times from the peak frequencies.

The dielectric spectra can be fit by assuming the various contributions are simply additive:

$$
\varepsilon^{*}(\omega)=\left(\sigma_{\mathrm{DC}} / i \omega \varepsilon_{0}\right)^{n}+\varepsilon_{\alpha}^{*}+\frac{\Delta \varepsilon_{\beta}}{\left(1+\left(i \omega \tau_{\mathrm{HN}}\right)^{a}\right)^{b}}+\varepsilon_{\infty}
$$


The first term on the right-hand side represents the conductivity due to mobile ionic impurities, with the exponent being nearly constant $(0.8 \leq n \leq 1)$. The last two terms are the Havriliak-Negami function used to describe the secondary relaxation, with $\Delta \varepsilon_{\mathrm{HN}}$ the dielectric strength, $\tau_{\mathrm{HN}}$ the relaxation time, the constants $a$ and $b$ shape parameters, and $\varepsilon_{\infty}$ the highfrequency limiting value of the dielectric constant. The primary $\alpha$-relaxation is fitted to the Kohlrausch-Williams-Watts (KWW) correlation function ${ }^{32,33}$

$$
\varepsilon_{\mathrm{KWW}}(t)=\exp \left[-\left(t / \tau_{\mathrm{KWW}}\right)^{\beta_{\mathrm{KWW}}}\right]
$$

with

$$
\varepsilon_{\alpha}^{*}=\Delta \varepsilon_{\alpha} \int_{0}^{\infty} \frac{\mathrm{d} \varepsilon_{\mathrm{KWW}}(t)}{\mathrm{d} t} \mathrm{e}^{-i \omega t} \mathrm{~d} t
$$

with $\beta_{\mathrm{KWW}}$ the stretch exponent and $\tau_{\mathrm{KWW}}$ the relaxation time. The relaxation times reported herein are defined as the reciprocal of the frequency of the maximum in the dispersion; thus, $\tau_{\alpha}<\tau_{\mathrm{KWW}}$ and $\tau_{\mathrm{JG}}<\tau_{\mathrm{HN}}$. The real and imaginary permittivities in Figure 1 were simultaneously fit to eq 3, with the obtained values of the relaxation times indicated.

When the peaks in the spectrum are not well separated, there is an alternative to eq 3 that accounts for the effect that structural relaxation may have on the JG process. ${ }^{34}$

$$
\varepsilon(t)=f_{\alpha} \varepsilon_{\mathrm{KWW}}(t)+\left(1-f_{\alpha}\right) \varepsilon_{\mathrm{KWW}}(t) \varepsilon_{\mathrm{HN}}(t)
$$

In this equation, $f_{\alpha}$ is the relative strength of the $\alpha$-process and $\varepsilon_{\mathrm{HN}}(t)$ is the Fourier transform of the Havriliak-Negami function. This equation is only applied to the dielectric loss, and fitting to the $\varepsilon^{\prime \prime}(\omega)$ in Figure 1 gives a curve that cannot be discerned from the best fit of eq 3 . The relaxation times for the two analyses differ by less than $10 \%$; for more widely separated peaks this difference will, of course, be less.

To interpret the high-pressure data in term of the mass density requires the equation of state (EOS) for the PMMA. From measurements of the specific volume as a function of temperature and pressure, the Tait EOS above the glass transition is obtained

$$
\begin{gathered}
V(T, P)=V_{0} \exp \left(\alpha_{0} T\right)\{1-0.0894 \\
\left.\ln \left[1+P /\left(b_{0} \exp \left(-b_{1} T\right)\right)\right]\right\}
\end{gathered}
$$

with $V_{0}=0.826 \pm 0.003 \mathrm{~mL} / \mathrm{g}, \alpha_{0}=(6.2 \pm 0.04) \times 10^{-4} \mathrm{C}^{-1}$, $b_{0}=235 \pm 2 \mathrm{MPa}$, and $b_{1}=(3.75 \pm 0.01) \times 10^{-3} \mathrm{C}^{-1}$. The dielectric strength for the $\alpha$ and JG processes are displayed in Figure 2 as a function of density. For both isobaric (upper) and isothermal (lower) conditions, the relaxation strengths approach the same value at the glass transition, with $\Delta \varepsilon_{\alpha}$ tending to zero for shorter $\tau_{\alpha}\left(\cong 10^{-5} \mathrm{~s}\right)$. This behavior has been described previously for poly(alkyl methacrylate)s due to temperature changes alone. ${ }^{35}$ The results in Figure 2 show that the relaxation strengths are connected to the dynamics; that is, the relative dielectric strength for the two processes depends on the magnitudes of $\tau_{\alpha}$ and $\tau_{\mathrm{JG}}$. According to the KirkwoodFrölich relation, ${ }^{36}$ the dielectric strength should increase with increasing density (higher dipole concentration) and decreasing temperature (stronger dipole correlations). The data in Figure 2 are consistent with this expectation, $\Delta \varepsilon_{\alpha}$ increasing more strongly with $\rho$ for cooling at constant pressure than for isothermal densification.

The relaxation times of the $\alpha$-process obtained by fitting eq 3 to the spectra are displayed as a function of density in Figure 3.

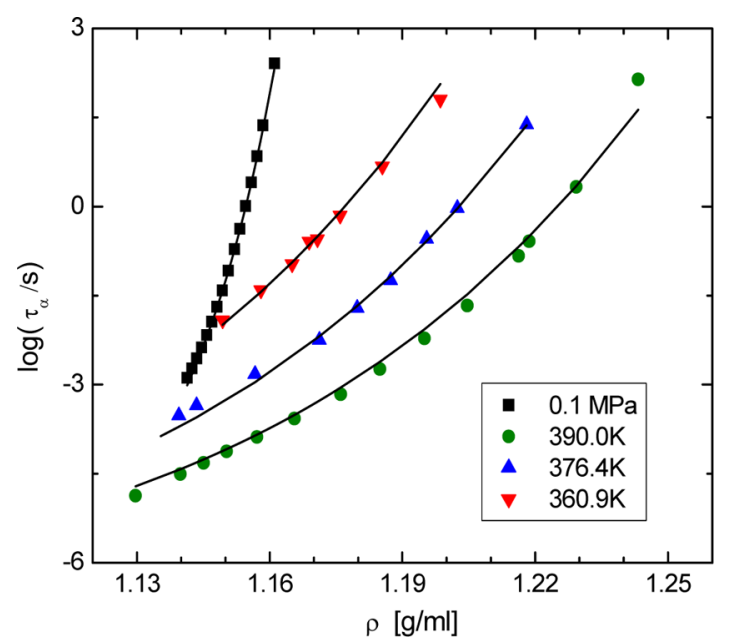

Figure 3. $\alpha$-Relaxation times versus density, along with fits to the modified Avramov equation (eq 8).

These data can be described by the modified Avramov equation $^{37}$

$$
\tau_{\alpha}(T, \rho)=\tau_{0} \exp \left[\left(\frac{\xi}{T \rho^{-\gamma}}\right)^{\phi}\right]
$$

where $\tau_{0}$ corresponds to the high $T$, low $\rho$ limit of $\tau_{\alpha}$, $\xi$ is related to the height and distribution of the energy barriers governing segmental motions, and $\phi$ is proportional to the isochoric heat capacity. The Avramov model relates changes of the relaxation time to changes of the entropy. Equation 8 was derived by expressing the entropy change as a function of density and temperature, with $\gamma$ identified as the Grüneisen constant. ${ }^{37,38}$ This equation satisfies the density scaling (eq 1).

From the fit of eq 8 to the $\tau_{\alpha}(T, \rho)$ (solid lines in Figure 3), the scaling parameter is determined, $\gamma=1.94 \pm 0.02$. (The values of the other parameters in eq 8 are $B=379 \pm 9 \mathrm{~kg}^{-\gamma}$ $\mathrm{mL}^{\gamma}, \log \left(\tau_{0} / \mathrm{s}\right)=-6.8 \pm 0.3$, and $\phi=7.5 \pm 0.4$.) Previously, we found that for a range of materials the parameters $\gamma$ and $\phi$ are anticorrelated and described approximately by the relation $\gamma \phi=$ $17.9 \pm 3.7$; for the present case, $\gamma \phi=14.5 \pm 0.9$, which is consistent within the error.

The relaxation times obtained from the spectra are plotted in the scaling form in Figure 4, with the exponent $\gamma$ determined from the fit of eq 8 to $\tau_{\alpha}$. The $\alpha$ relaxation times superpose well over 6 decades. Included in the figure are the JG relaxation times, which clearly deviate from density scaling. Given the narrow span of the $\tau_{\mathrm{JG}}$, density scaling could be achieved using a different value of $\gamma(\approx 1.1 \pm 0.1)$; however, $\tau_{\alpha}$ and $\tau_{\mathrm{JG}}$ do not superpose for the same value of $\gamma$.

Corroboration of this finding can be obtained by comparing the positions of the two dispersions. Thus, as shown in Figure 5 , we fit three loss spectra to eq 3 using constant values of $\beta_{\mathrm{KWW}}=0.38$ and $\log \left(\tau_{\alpha} / \mathrm{s}\right)=-1.749$. The obtained $\tau_{\mathrm{JG}}$, listed in the figure, increase significantly with increasing temperature. This variation in $\tau_{\mathrm{JG}}$ for fixed $\tau_{\alpha}$ means the two relaxation processes do not conform to eq 1 for a common value of $\gamma$. This also implies deviation from eq 2 of the coupling model. In Figure 6 the same analysis is carried out for a PMMA of lower molecular $\left(M_{\mathrm{w}}=402 \mathrm{Da}\right)$, which has substantially greater separation of the $\alpha$ and JG dispersions. For essentially constant $\tau_{\alpha}$, the JG relaxation times differ by 1.3 decades, further 


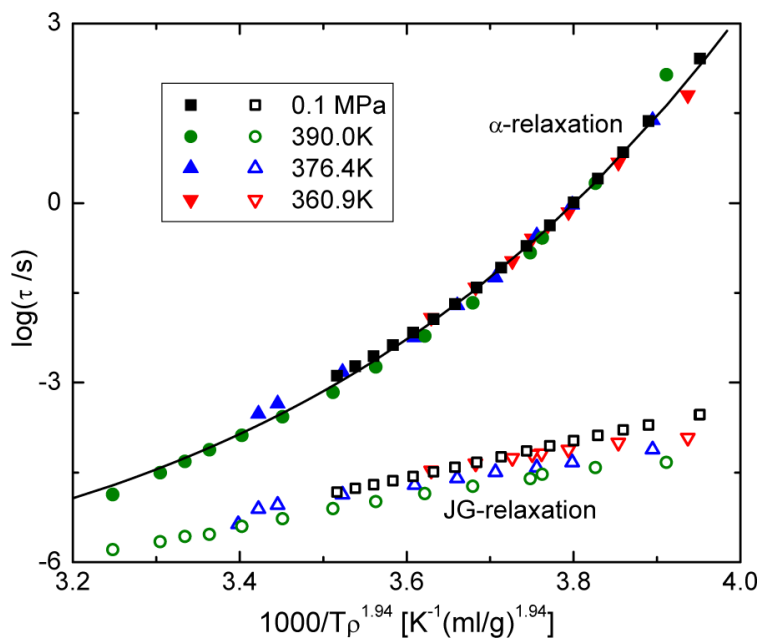

Figure 4. Density scaling plots of the relaxation times for the $\alpha$ (filled symbols) and JG (open symbols) processes. Only the former superpose; the solid line is eq 8 with $\gamma=1.94$ (which is also shown in Figure 3).

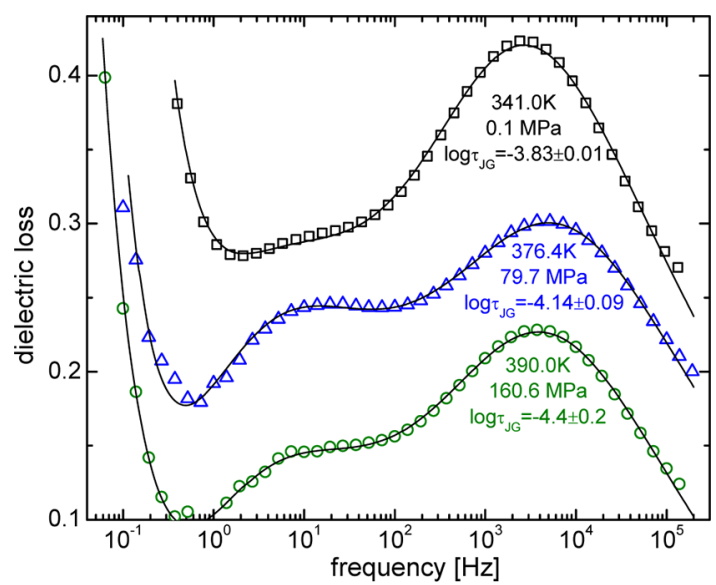

Figure 5. Fitted spectra for which $\tau_{\alpha}$ is constant (=17.8 ms); as indicated, $\tau_{\mathrm{JG}}$ increases with increasing temperature. There is also a change in the relative dielectric strength of the two processes. The spectra were displaced vertically for clarity.

supporting our result that the JG process does not density scale for the same $\gamma$ that superposes the $\alpha$ relaxation times.

The scaling exponent for PMMA, $\gamma=1.94$, is at the low end of the range reported for van der Waals liquids and polymers, $1.8 \leq \gamma \leq 8.5$. $^{4-6}$ The inference is that the segmental dynamics of PMMA is governed primarily by temperature, with a relatively weak effect of density. As seen in Table 1 , which includes prior results ${ }^{39,40}$ on other PMMA samples, the scaling exponent for PMMA varies inversely with molecular weight, consistent with the general trend that polymers have smaller $\gamma$ than the values for molecular liquids. Density effects on the dynamics are weaker for polymers than small molecules because of the influence of the intrachain potential on the dynamics of polymers. ${ }^{5,12}$ Thus, large density scaling exponents are only found for polymers with very flexible backbones (e.g., polysiloxanes $^{41}$ ).

In addition to serving as a measure of the relative influence of thermal energy and density on the dynamics, $\gamma$ has another interpretation. From molecular dynamics simulations, the scaling exponent for Lennard-Jones particles was found to equal one-third the slope of the intermolecular potential in the

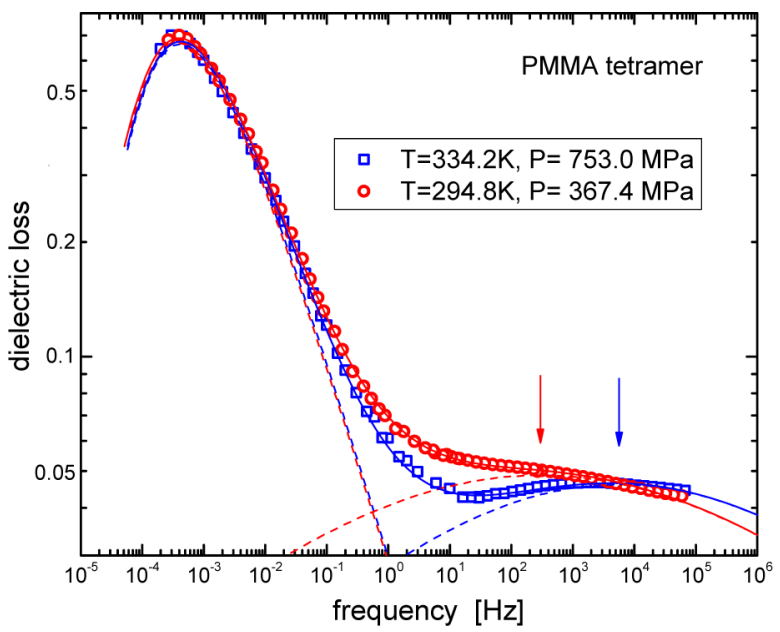

Figure 6. Loss spectra of a lower molecular weight PMMA $\left(M_{\mathrm{w}}=402\right.$ $\mathrm{Da})$ at two different temperatures and pressures, for which the stretch exponent in eq 4 is the same, $\beta_{\mathrm{KWW}}=0.52 \pm 0.01$. The lower temperature spectrum was shifted by 0.05 decades to lower frequency and by a factor of 0.93 in intensity, in order to superpose the $\alpha$ peaks. The vertical arrows denote the position of the maximum in the JG peaks; their frequencies differ by 1.3 decades, despite the isochronal superpositioning of the $\alpha$ dispersions. Solid lines are the fits of eq 3, with the individual contributions indicated by the dotted lines.

Table 1. Scaling Exponent Dependence on Molecular Weight of PMMA

\begin{tabular}{rll}
$M_{\mathrm{w}}(\mathrm{Da})$ & $\gamma($ eq 1$)$ & reference \\
302 & 3.7 & 39 \\
402 & 3.2 & 39 \\
1040 & 2.8 & 39 \\
1970 & 1.94 & this work \\
150000 & 1.8 & 40 \\
\hline
\end{tabular}

region between the distance of closest approach between particles and the half-maximum of the pair distribution function. ${ }^{9}$ From this fact and notwithstanding that eq 1 is invariably applied to $\tau_{\alpha}$, Ngai et al. ${ }^{22}$ surmised that the scaling property originates in the JG relaxation, rather than the $\alpha$ process. This conclusion is undermined by the present finding that different scaling exponents are required to superpose $\tau_{\alpha}$ and $\tau_{\mathrm{JG}}$. The argument in ref 22 is that the relevant intermolecular distance over which $\gamma$ is equivalent to the steepness of the potential is much shorter than the length scale of the $\alpha$-relaxation. Although this length scale is a measure of the spatial extent of the $\alpha$-process, this does not mean that over the course of structural relaxation intermolecular distances attain such large lengths. Rather, the particular pair contacts change as the relaxing species encounter different near neighbors; however, the intermolecular distances governing the potential remain smaller than the $\alpha$-relaxation length scale. Thus, the reported connection ${ }^{1,9,11}$ between the magnitude of $\gamma$ and the slope of the intermolecular potential is consistent with density scaling originating with the $\alpha$-process.

\section{SUMMARY}

It was recently proposed that density scaling of the structural relaxation in liquids and polymers originates from the JG relaxation. This leads to the expectation that $\tau_{\alpha}$ and $\tau_{\mathrm{JG}}$ should superpose according to eq 1 for the same value of $\gamma$. A rigorous test of this prediction is difficult, since the JG relaxation is 
usually measured below the glass transition temperature, whereby the $\alpha$ relaxation is too slow for accurate characterization. (It is presently unknown if scaling of the $\alpha$ relaxation is valid in the glassy state.) Moreover, $\tau_{\mathrm{JG}}$ for a glass is not a unique function of density, as seen in aging experiments ${ }^{42,43}$ and for pressure-densified glasses; ${ }^{23-25}$ these experiments have shown that increasing the density of a glass can cause the JG process to become either faster or slower. Such behavior renders untenable the hypothesis that $\tau_{\mathrm{JG}}$ conforms to density scaling in equivalent fashion as $\tau_{\alpha}$.

Thus, to test the hypothesis that $\tau_{\alpha}$ and $\tau_{\mathrm{JG}}$ follow eq 1 with a common $\gamma$, we carried out measurements on a material for which both processes can be measured above $T_{\mathrm{g}}$. We find that while good superpositioning of $\tau_{\alpha}$ for low molecular weight PMMA can be achieved, $\tau_{\mathrm{JG}}$ deviate from the scaling. At constant $\tau_{\alpha}$ higher pressures are associated with greater separation in frequency of the two relaxation peaks. Given the conformance of PMMA to isochronal superpositioning $\left(\beta_{\mathrm{KWW}}\right.$ constant for constant $\left.\tau_{\alpha}^{29,30}\right)$, this result indicates a departure from the $\tau_{\mathrm{JG}}$ calculated from eq 2 .

\section{AUTHOR INFORMATION}

\section{Corresponding Author}

*E-mail roland@nrl.navy.mil (C.M.R.), Riccardo.Casalini@nrl. navy.mil (R.C.).

\section{Notes}

The authors declare no competing financial interest.

\section{ACKNOWLEDGMENTS}

We thank D. Fragiadakis for many helpful discussions and for the Grafity software used to carry out the analyses herein. A. R. Ellis assisted with the PVT measurements. This work was supported by the Office of Naval Research.

\section{REFERENCES}

(1) Casalini, R.; Roland, C. M. Phys. Rev. E 2004, 69, 062501.

(2) Alba-Simionesco, C.; Cailliaux, A.; Alegria, A.; Tarjus, G. Europhys. Lett. 2004, 68, 58.

(3) Dreyfus, C.; Le Grand, A.; Gapinski, J.; Steffen, W.; Patkowski, A.

Eur. J. Phys. 2004, 42, 309.

(4) Roland, C. M. Macromolecules 2010, 43, 7875.

(5) Roland, C. M. Viscoelastic Behavior of Rubbery Materials; Oxford Univerasity Press: Oxford, UK, 2011.

(6) Roland, C. M.; Hensel-Bielowka, S.; Paluch, M.; Casalini, R. Rep. Prog. Phys. 2005, 68, 1405.

(7) Pensado, A. S.; Padua, A. A. H.; Comunas, M. J. P.; Fernandez, J. J. Phys. Chem. B 2008, 112, 5563.

(8) Lopez, E. R.; Pensado, A. S.; Comunas, M. J. P.; Padua, A. A. H.; Fernandez, J.; Harris, K. R. J. Chem. Phys. 2011, 134, 144507.

(9) Coslovich, D.; Roland, C. M. J. Phys. Chem. B 2008, 112, 1329.

(10) Schrøder, T. B.; Pedersen, U. R.; Bailey, N. P.; Toxvaerd, S.; Dyre, J. C. Phys. Rev. E 2009, 80, 041502.

(11) Fragiadakis, D.; Roland, C. M. J. Chem. Phys. 2011, 134, 044504.

(12) Roland, C. M.; Bair, S.; Casalini, R. J. Chem. Phys. 2006, 125, 124508.

(13) Coslovich, D.; Roland, C. M. J. Chem. Phys. 2009, 130, 014508.

(14) Gundermann, D.; Pedersen, U. R.; Hecksher, T.; Bailey, N.; Jakobsen, B.; Christensen, T.; Olsen, N. B.; Schrøder, T. B.; Fragiadakis, D.; Casalini, R.; Roland, C. M.; Dyre, J. P.; Niss, K. Nat. Phys. 2011, 7, 816.

(15) Fragiadakis, D.; Casalini, R.; Bogoslovov, R. B.; Robertson, C. G.; Roland, C. M. Macromolecules 2011, 44, 1149.

(16) Casalini, R.; Roland, C. M. Macromolecules 2005, 38, 1779.

(17) Roland, C. M.; Paluch, M.; Casalini, R. J. Polym. Sci., Part B: Polym. Phys. 2004, 42, 4313.
(18) Doi, M.; Edwards, S. F. The Theory of Polymer Dynamics; Clarendon: Oxford, UK, 1986.

(19) Watanabe, H. Prog. Polym. Sci. 1999, 24, 1253.

(20) Ngai, K. L.; Casalini, R.; Roland, C. M. Macromolecules 2005, 38, 4363.

(21) Ngai, K. L. J. Phys.: Condens. Matter 2003, 15, S1107.

(22) Ngai, K. L.; Habasaki, J.; Prevosto, D.; Capaccioli, S.; Paluch, M. J. Chem. Phys. 2012, 137, 034511.

(23) Casalini, R.; Roland, C. M. J. Non-Cryst. Solids 2011, 357, 282.

(24) Casalini, R.; Roland, C. M. J. Chem. Phys. 2009, 131, 114501.

(25) Casalini, R.; Roland, C. M. Phys. Rev. Lett. 2009, 102, 035701.

(26) Ngai, K. L. Comment Solid State Phys. 1979, 9, 127.

(27) Ngai, K. L. J. Chem. Phys. 1998, 109, 6982.

(28) McCrum, N. G.; Read, B. E.; Williams, G. Anelastic and Dielectric Effects in Polymeric Solids; Wiley: New York, 1967.

(29) Roland, C. M.; Casalini, R.; M. Paluch, M. Chem. Phys. Lett. 2003, 367, 259.

(30) Ngai, K. L.; Casalini, R.; Capaccioli, S.; Paluch, M.; Roland, C. M. J. Phys. Chem. B 2005, 109, 17356.

(31) Schmidt-Rohr, K.; Kulik, A. S.; Beckham, H. W.; Ohlemacher, A.; Pawelzik, U.; Boeffel, C.; Spiess, H. W. Macromolecules 1994, 27, 4733.

(32) Kohlrausch, R. Ann. Phys. 1847, 12, 393.

(33) Williams, G.; Watts, D. C. Trans. Faraday Soc. 1970, 66, 80.

(34) Williams, G. Adv. Polym. Sci. 1979, 33, 60.

(35) Garwe, F.; Schonhals, A.; Lockwenz, H.; Beiner, M.; Schroter, K.; Donth, E. Macromolecules 1996, 29, 247.

(36) Broadband Dielectric Spectroscopy; Kremer, F., Schonhals, A.; Eds.; Springer: New York, 2002.

(37) Casalini, R.; Mohanty, U.; Roland, C. M. J. Chem. Phys. 2006, $125,014505$.

(38) Casalini, R.; Roland, C. M. J. Non-Cryst. Solids 2007, 353, 3936.

(39) Casalini, R.; Roland, C. M.; Capaccioli, S. J. Chem. Phys. 2007, $126,184903$.

(40) Theobald, S.; Pechhold, W.; Stoll, B. Polymer 2001, 42, 289.

(41) Casalini, R.; Roland, C. M. Colloid Polym. Sci. 2004, 283, 107.

(42) Schneider, U.; Brand, R.; Lunkenheimer, P.; Loidl, A. Phys. Rev. Lett. 2000, 84, 5560.

(43) Olsen, N. B. J. Non-Cryst. Solids 1998, 235-237, 399. 